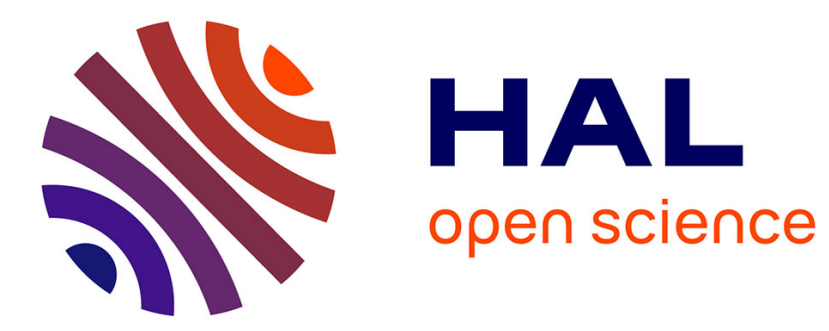

\title{
Gattung und Geschlecht bei Sophie von La Roche und Maria Anna Sager
}

Helga Meise

\section{To cite this version:}

Helga Meise. Gattung und Geschlecht bei Sophie von La Roche und Maria Anna Sager. Seminar: A Journal of Germanic Studies, 2013, The Eighteenth-Century Novel as Media Event, 49 (2), pp.131-147. 10.3138/sem.49.2.131 . hal-02475119

\section{HAL Id: hal-02475119 \\ https://hal.univ-reims.fr/hal-02475119}

Submitted on 12 Nov 2021

HAL is a multi-disciplinary open access archive for the deposit and dissemination of scientific research documents, whether they are published or not. The documents may come from teaching and research institutions in France or abroad, or from public or private research centers.
L'archive ouverte pluridisciplinaire HAL, est destinée au dépôt et à la diffusion de documents scientifiques de niveau recherche, publiés ou non, émanant des établissements d'enseignement et de recherche français ou étrangers, des laboratoires publics ou privés.

\section{(c)(1)}

Distributed under a Creative Commons Attribution| 4.0 International License 


\section{Gattung und Geschlecht bei Sophie von La Roche und Maria Anna Sager}

Helga Meise

Université de Reims Champagne-Ardenne

Sophie von La Roche (1730-1807) und Maria Anna Sager (1719-1805) sind die ersten Frauen der deutschsprachigen Aufklärung, die Romane veröffentlichten. La Roches Geschichte des Fräuleins von Sternheim. Von einer Freundin derselben aus Original-Papieren und andern zuverläßigen Quellen gezogen erscheint 1771 anonym bei Weidmanns Erben und Reich in Leipzig; als Herausgeber figuriert Christoph Martin Wieland (1733-1813). Sagers Die verwechselten Töchter, eine wahrhafte Geschichte in Briefen entworfen von einem Frauenzimmer und Karolinens Tagebuch ohne ausserordentliche Handlungen oder gerade so viel als gar keine kommen 1771 und 1774 bei Wolfgang Gerle in Prag heraus. Der erste Roman erscheint anonym, der zweite unter dem Kryptonym „M. A. S.“ Dass sich dahinter Maria Anna Sager verbirgt, konnte ich anhand von zeitgenössischen Quellen zeigen; dass die Autorin selbst in ihren letzten Lebensjahren mit dem Namen „Sager" unterschrieb (Cornova 55), legt nahe, diese Schreibweise anstelle des in der Forschung geläufigen „Sagar“ zu verwenden.

Jeder Roman thematisiert das Verhältnis von Gattung und Geschlecht: er stellt die Geschichte weiblicher Titelfiguren in den Mittelpunkt und fragt gleichzeitig nach der Form der Darstellung. Jeder bringt damit eine zentrale „Denkfigur“ (Fleig und Meise 159) der zeitgenössischen Gattungspoetik ins Spiel. Gemeint ist die Affinität, ja der vermeintlich unmittelbar gegebene Zusammenhang von literarischer Gattung und weiblichem Geschlecht, den Christian Fürchtegott Gellerts (1715-1769) „Natürlichkeitspostulat“, aufgestellt 1742 und 1752 für die Abfassung von Briefen, in den Blick gerückt hatte: „Es ist offensichtlich, daß die Frau von Gellert mit dem natürlichen Briefstil identifiziert wird. Und Gellert ist genau der erste, der diese Verbindung herstellt.“ (Nörtemann 22) Die Leichtigkeit, mit der Frauen „,natürlich, aufgeweckt, naiv und überzeugend ... schreiben“ (Nörtemann 21), schien sie nicht nur zur Abfassung von Briefen, sondern auch zu der von Briefromanen und Romanen zu prädestinieren - verfügten sie doch infolge ihrer nur beschränkten Bildungs- und Ausbildungsmöglichkeiten über den unmittelbaren Zugang nicht nur zu ihren Empfindungen und Gefühlen, sondern auch zu deren Ausdruck und damit auch über das Rüstzeug für die neuen Gattungen Brief, Briefroman und Roman. Johann Georg Sulzer (1720-1779) überträgt diese Überlegungen auf die Lyrik; seine Vorrede von 1764 zu den Gedichten von Anna Louisa Karsch (1722-1791) lobt, dass bei dieser Dichterin „die Natur durch die Begeisterung würket“, und zwar „Ohne Vorsatz, ohne Kunst und Unterricht“ (IX). Wieland wendet sie in der Vorrede zur Sternheim auf den Roman und die weibliche Autorschaft an. Aber schon Friedrich von Blanckenburgs (1744-1796) Versuch über den Roman von 1774, die erste romantheoretische Schrift des deutschsprachigen Raums, die den Roman auf die Darstellung der ,inneren Geschichte eines Menschen“ verpflichtet, veranschaulicht diese Bestimmung an Wielands Agathon von 1767 (see e.g. Schlimmer, Romanpoetik 47) und damit an einer männlichen Titelfigur. Dies kommt einer Festlegung und einer Instrumentalisierung des Romans gleich. Er wird, wie Schlimmer gezeigt hat, zum „Bildungsmittel“ für „die Bewältigung der alltäglichen Lebenspraxis“ und den dazu erforderlichen Umgang mit 
„Empfindungen und Gefühlen“ („Roman als Erziehungsanstalt“ 220): Er dient „,zur Unterhaltung, zur Verbesserung, zur Vervollkommnung des menschlichen Geschlechts' [Blanckenburg, H. M.]. Der Roman wird zu einer Erziehungsanstalt für Leser.“ (220)

Damit bahnt Blanckenburgs Versuch dem Aufstieg des Bildungsromans den Weg. Mit „Geschlecht" meint er indes nur das männliche Geschlecht: Individuen männlichen Geschlechts vermögen eine Entwicklung zu durchlaufen, wie Goethes Wilhelm Meister in den Lehrjahren (1795) programmatisch formulieren wird: „mich selbst, ganz wie ich da bin, auszubilden, das war dunkel von Jugend auf mein Wunsch und meine Absicht." (290) Blanckenburgs „Fixierung“ auf den Romantypus Entwicklungsroman, so Schlimmer („Roman als Erziehungsanstalt“ 220), setzt mit der Begründung des Romans als literarischer Gattung auch die Unterscheidung von literarisch wertvoller und bloß unterhaltender Literatur ins Werk. Mit dem ausgehenden 18. Jahrhundert wird „der Bildungsroman zur einzig anerkannten Form der Gattung erklärt" (220), andere Romantypen werden abgewertet und aus dem sich formierenden Kanon anerkannter Texte verdrängt, selbst wenn diesen wie auch im Fall der von Frauen verfassten Romane, ein eigener Typus - eben der „Frauenroman“ zugestanden wird, wie Schlimmer festhält (221). Diese wenigen Belege zeigen, dass Fragen nach dem Geschlecht den gattungspoetischen Diskussionen um den Roman von Beginn an eingeschrieben sind, unabhängig davon, ob das Geschlecht explizit oder implizit verhandelt wird. Der vermeintlich genuin literarische Gattungsdiskurs wird verstärkt durch Diskussionen außerhalb des literarischen Feldes, die ihrerseits Fragen nach dem Geschlecht aufwerfen, aber das „menschliche Geschlecht” (Blanckenburg) nach männlich und weiblich differenzieren. Schon seit dem Ende des 17. Jahrhunderts hatten verschiedene Strömungen der sich ausbildenden Naturwissenschaften zur „,Entdeckung' des Geschlechtsgegensatzes im Leib“ geführt und den „Geschlechtsunterschied“ (Laqueur 172) als biologische Tatsache beschrieben. Die Rezeption von Jean-Jacques Rousseaus Emile ou De l'éducation (1762) etabliert den Diskurs über die Unterschiede zwischen den Geschlechtern auch außerhalb der Naturwissenschaften. Sie bringt die Rede von den von der „Natur“ gewollten „Geschlechtscharakteren“ (Hausen) hervor und stellt damit eine ebenso begründete wie griffige Definition zur Unterscheidung der Geschlechter bereit. Reformvorhaben zur Mädchen- und Frauenbildung im Umkreis der Philanthropen, in der Philosophie und in den Wissenschaften vom Menschen knüpfen daran an; die neu gefundene „Ordnung der Geschlechter" (Honegger) sollte die Moderne bis ins 20. Jahrhundert hinein prägen.

Um so mehr frappiert, dass die ersten Romane von Frauen im deutschsprachigen Raum das Verhältnis von Gattung und Geschlecht vollkommen unterschiedlich denken. Wie Vorreden, Raumsemantik und Darstellungsformen im Vergleich zeigen, erzeugt La Roches Sternheim Gattung und Geschlecht mit den überkommenen Mitteln des Briefromans. Sagers Die verwechselten Töchter und Karolinens Tagebuch hingegen stürzen Gattung und Geschlecht in eine mise en abyme, die beide Begriffe ohne Ende aushebelt, um gleichzeitig auf die Schaffung neuer Romane zu verweisen.

\section{Die Vorreden}

Wielands Vorrede zur Sternheim gilt zu Recht als „Schlüsseltext für die Literaturdiskussion zu jener Zeit" (Kohl 27). Sie ist, auch im Blick auf Wielands Vorreden zu seinen eigenen Romanen (see e.g. Wirth; Frick), ein Bravourstück, übersetzt sie doch die Denkfigur von der Affinität von Gattung und Geschlecht und das Denken des Geschlechtsunterschieds ineinander und installiert im selben Atemzug den männlichen Herausgeber als Meister (Baldwin; Loster-Schneider; Jirku). Der Text präsentiert sich zum einen als eine sich immer weiter steigernde Lobrede des Herausgebers auf die Vorzüge des ihm vorliegenden Romans. 
„Einer der seltensten“, so die Schlussfolgerung, bestehe in der „völligen Individualisierung des Charakters unsrer Heldin“ (15), der direkt aus der Natur ,gearbeitet“ (15) sei: sie habe „ungeachtet die Vorteile ihrer Erziehung bei aller Gelegenheit hervorschimmern, dennoch ihren Geschmack und ihre Art zu denken, zu reden und zu handeln, mehr der Natur und ihren eigenen Erfahrungen und Bemerkungen, als dem Unterricht und der Nachahmung zu danken“ (14). Zum anderen wird der Roman der ,moralischen Dichtung“ zugeschlagen, die nicht auf „künstliche Form“, sondern auf „moralische Nützlichkeit“ (14) ziele. Aufwertung und Abwertung sind eins. Das ambivalente Verhältnis von Vorzug und Mangel kennzeichnet ebenso die Figur des Autors bzw. der Autorin. In Analogie zur Charakterisierung der Titelfigur und der didaktischen Funktion des Romans wird die Autorin auf die Position der moralischen Dichterin festgeschrieben. Sie habe nur auf den Inhalt, nicht aber die Form geachtet (13), so dass sie hinter dem Nutzen, den ihr Werk verkörpert, verschwindet: „Doch der liebste Wunsch unsrer Heldin ist nicht der Wunsch der Eitelkeit; nützlich zu sein, wünscht sie; Gutes will sie tun; und Gutes wird sie tun, und dadurch den Schritt rechtfertigen, den ich gewaget habe, sie ohne Vorwissen und Erlaubnis ihrer liebenswürdigen Urheberin in die Welt einzuführen. Ich bin, usw. Der Herausgeber“ (17, Hervorhebung im Original). So wie „hier, wo die Natur gearbeitet“ (15) habe, habe die „Urheberin“ auch nie daran gedacht, „für die Welt zu schreiben oder ein Werk der Kunst hervorzubringen“ (13). Was ihr Schreiben als Vorzug auszeichnet, schließt die Autorin definitiv aus den „Autors-Künsten“ (14) aus - sie sei weder „Schriftstellerin von Profession“ (15) noch halte sie sich an die „Regeln der Kunst“ (14).

Im Gegenzug zu der „,ölligen Individualisierung“, die die weibliche Titelfigur mit allen ihren Tugenden unverkennbar und anders macht und ihre Autorin auf eine Rolle fixiert, behält sich der Herausgeber selbst eine große Beweglichkeit, ja Verwandlungsfähigkeit vor. Die unterschiedlichen Rollen, in die er nach und nach schlüpft, sind ihrerseits in sich gesteigert. Er ist zunächst der Freund, dem eine „Freundin“ (10) ihren handgeschriebenen Text anvertraut: „,nur für Sie und mich“ (9). Entsprechend kleidet er die Vorrede in die Form des Briefes, der mit der Freundin ,nur' private Dinge verhandelt. Aber der Freund wandelt sich zum Leser, der den Text „liebt“ (11), zum „Kunstrichter“ (13), der ihn „nach den Regeln der Kunst“ (14) und dem „Geschmacke der Weltleute“ (15) beurteilt, und schließlich zu seinem „Herausgeber“ (17). Die Rolle des Herausgebers hebt alle übrigen Rollen in sich auf und verschafft diesem so die Autorität, über das ihm anvertraute Werk „eigenmächtig“ (13) zu verfügen, sogar über seine eingestandene „Verräterei“ (9) hinweg. Gleichzeitig trennt ein Schnitt die Rollen voneinander: „Bei aller Wärme meines Herzens blieb doch mein Kopf kalt genug, um alles in Betrachtung zu ziehen, was vermögend schien, mich von meinem Vorhaben [der Veröffentlichung, H. M.] abzuhalten“ (12). Die Liebe weicht der kalten Untersuchung, der vorurteilsfreien (13) Analyse durch den „Kunstrichter“, „Kenner“ und Weltmann (13). Nur auf der Grundlage seines sachlichen Urteils kann der Text aus der Privatheit in eine Öffentlichkeit wechseln, in der „Auspfeifer“ (13) jede Neuerscheinung begierig erwarten.

Wielands Vorrede will schildern, ,wie der Gedanke, Sie in eine Schriftstellerin zu verwandeln, in mir entstanden ist" (10). Tatsächlich beschreibt sie die einzelnen Stationen von der „Handschrift” (9) über „eine schöne Abschrift von Ihrem Manuskripte“ (10) und „Copeyen für alle unsere Freunde und Freundinnen“ (11), mithin ,eine große Menge“ (11), zur „gedruckten Copey“ (9) -, koppelt aber jede von ihnen an die Rolle, die der Mann übernimmt. In dem Maße, in dem die Schriftstellerin modelliert wird, verschwindet die „Urheberin“ (17); der Roman entsteht und die Figur des Herausgebers. Dieser verrät unwillkürlich sein Geschlecht, die Spannung zwischen Begehren und Unterwerfung, die ihn umtreibt: Als Freund wird er zum „Pflegevater“ (Kohl, 30), als Leser sieht er mit „süßester 
Wollust" (11) die Eigenschaften der Titelfigur in seinen eigenen Töchtern nachgebildet. Gleichzeitig bleibt die „Urheberin“ seinem Urteil unterworfen. Es ist kein Zufall, dass seine Rollen alle Instanzen des literarischen Systems abdecken - der Leser, Kritiker und Herausgeber entpuppt sich als längst bekannter Autor.

Dass jeder Rollenwechsel des Mannes die Festlegung des Werkes und seiner Autorin auf geschlechtsspezifische - weibliche - Merkmale zementiert, belegt noch das Lesepublikum, das jeweils anvisiert wird. Als Freund und Leser will er nicht nur der eigenen Tochter, sondern ,allen tugendhaften Müttern, allen liebenswürdigen Töchtern unserer Nation ein Geschenk mit einem Werke machen, welches mir geschickt schien, Weisheit und Tugend die einzigen großen Vorzüge der Menschheit, die einzigen Quellen einer wahren Glückseligkeit - unter Ihrem Geschlechte, und selbst unter dem meinigen, zu befördern" (10) (see e.g. Schön). Als Kritiker, der sich aufgrund des Inhalts über die formalen Unvollkommenheiten des Werkes hinwegsetzt, nimmt er die Meinung seiner Kollegen vorweg: „Kurz, ich habe eine so gute Meinung von der feinen Empfindung der Kunstrichter, daß ich ihnen zutraue, sie werden die Mängel . . . mit so vielen Schönheiten verwebt finden . . “ (15). Und als Weltmann meint er zu wissen, dass sie „eben darum, weil sie eine Erscheinung ist, unter dem Namen der liebenswürdigen Grillenfängerin ansehnliche Eroberungen sollte machen können“ (16).

Die Effekte dieser Vorrede setzen sich in den Anmerkungen des Herausgebers im Text fort (Baldwin 111; Jirku 157). Eine Autorin, so ist klar, bleibt auf die männliche Autorität angewiesen. Der Frau wird zwar aufgrund ihrer Natur sprachliche Kreativität und „Einbildungskraft“ $(9,16)$ zugestanden; aber im selben Moment wird sie auf ihre besondere Natur beschränkt und aus dem literarischen Feld ausgeschlossen, da sie nicht über die zur Hervorbringung von Kunstwerken nötigen Regeln verfüge (Loster-Schneider 88; Jirku 155).

Der Kontrast zu Sagers Vorreden könnte nicht größer sein (Baldwin 143; Jirku 159). Jedes Mal ergreift die Autorin selbst das Wort, jedes Mal stellt sie die Veröffentlichungspraktiken, ja die Zwänge bloß, auf die eine Autorin trifft. In Die verwechselten Töchter, eine wahrhafte Geschichte, in Briefen entworfen von einem Frauenzimmer, adressiert an „Meine Leser!“ (unpag.), bedient sie zuerst die gängigen Erwartungen mit der Versicherung, dass „die Geschichte, welche diese Briefe enthalten, wahrhaft ist; und daß ich die Namen bloß nach meiner Fantasie dazu hergegeben habe“ (unpag.). Aber gleich anschließend greift sie die Institution der Vorrede direkt an:

\footnotetext{
... ich glaube aber, daß ich vorher [vor der Lektüre des Textes, H. M.] noch auf die kräftigst mögliche Weise dagegen protestieren muß, daß man das, was ich hier schreibe, nicht als eine Vorrede ansehe. Die Hand eines Frauenzimmers ist viel zu zart, als daß sie, wie die männlichen Bücherschreiber, in dem Wust einer sophistischen Gelehrsamkeit herumwühlen sollte, um wie sie öfters über dunkele Stellen, eines dunklen Werkes, noch dunklere Erklärungen zu geben - Nein, o nein, deshalben schreibe ich diese Zeilen nicht . . . (unpag.).
}

Die Beweggründe zeitgenössischer Autoren - „Schreibsucht, Ehrgeiz nach der Autorschaft, und so weiter " (unpag.) - werden zwar benannt, aber nur, um gängige Rechtfertigungstopoi wie die vom Drängen des Verlegers oder der Unterwerfung unter die „Herren Kunstrichter“ als Camouflage zu entlarven. Ihr Hinweis auf die eigene Geschlechtszugehörigkeit zieht eine weitere Ebene ein: „Ich würde durch dergleichen Entschuldigungen mein Geschlecht' zu sehr herabsetzen, welches ...“ (unpag.). Sie verwahrt sich gegen eine derartige „Beleidigung“ der weiblichen Eitelkeit, behauptet die Bedeutungslosigkeit des „Beyfalls der Kunstrichter“ für ihr Geschlecht und fordert: „Ich kann meinem Geschlechte nicht so viel von seinen Gerechtsamen vergeben; die finstern Gelehrten nennen es selbst das Schöne, folglich müssen 
sie auch alles, was von uns kommt, ohne Ausnahme für schön erkennen, und hierunter ist meine gegenwärtige Arbeit auch mit verstanden." (unpag.) Während Wieland das „Privilegium der Damen, welche keine Schriftstellerinnen von Profession sind“ (15) ausdrücklich fallen läßt, um die Sternheim als Werk der Natur ausgeben zu können, nimmt Sagers Töchter die „Gerechtsamen“ des weiblichen Geschlechts bewußt und ,ironisch“ (Jirku 159; Heuser 65) zugleich zur Demonstration des eigenen Anspruches auf Autorschaft in Dienst. Es folgt eine letzte Volte: „.. . ich rathe es Ihnen noch einmal, meine Herren Leser! tadeln sie nichts an meinem Werkgen; den Kopf sollen sie nicht einmal darüber schütteln! oder ich werde mich mit einer Fortsetzung an ihnen rächen.“ (unpag.)

Der zweite Roman Karolinens Tagebuch, ohne ausserordentliche Handlungen oder gerade so viel als gar keine führt dieses Spiel mit der Lesererwartung weiter. Die nun dezidiert benannte „Vorrede“ kommentiert den Titel: „Wie meine lieben Leserinnen, Sie haben das Titelblatt gelesen, und wollen doch in dem Buche noch weiter umblättern; hat es sie nicht gewarnet, das Werkchen gleich wieder wegzulegen; wollen sie mit Nichts - und was ist ein Tagebuch ohne ausserordentliche Handlungen anderst - ihre Zeit versplittern?“ (unpag.) Angaben zur Autorin untergraben den Stellenwert des vorliegenden Romans weiter. Ihre böhmische Herkunft macht sie verdächtig, ebenso der Umstand, dass eine Fremde „ein Buch“ schreibt und ihre Leserinnen offen davor warnt. Andererseits besteht die Autorin auf der Gleichheit mit ihren Leserinnen, ihrer gemeinsamen Erfahrung als Angehörige ein- und desselben Geschlechts: „,vielleicht aber kenne ich mein Geschlecht besser, und verstehe es, ihre Neugier zu reitzen: so schliesse ich nach mir selbst. Denn je mehr man mich von etwas abhält, desto begieriger bin ich darauf. Aber nur still! Das bleibt unter uns Frauenzimmern im Vertrauen gesagt." (unpag.)

Der Titel des ersten Romans sowie das Spiel der Vorreden mit den Vorrechten des weiblichen Geschlechts, dem „Nichts“ und der Lust auf literarische Produktivität stellen die weibliche Autorschaft ostentativ aus. Dass die Anrede von „Meine Leser!“ (unpag.) im ersten zu „Meine lieben Leserinnen“ (unpag.) im zweiten Roman wechselt, ist programmatisch. Die Autorin und ihre Romane entziehen sich damit von Anfang an und auf den ersten Blick der „Systemfalle“ (Loster-Schneider) des Zusammenhangs von Gattung, Geschlecht und Autorschaft, in die Wielands Vorrede La Roche und die Sternheim manövriert.

\section{2. „Das Mädchen macht eine ganz neue Gattung von Charakter aus“: Weibliches Geschlecht und Raumsemantik}

Wie stellen die Romane selbst Geschlecht dar? Die Geschichte des Fräuleins von Sternheim erzählt, wie die junge Sophie von Sternheim als verwaiste Landadelige zu ihrer Tante an den Hof in D. übersiedeln muß. Als sie dem Fürsten als Mätresse zugespielt werden soll, rettet sie sich durch die Flucht und die Eheschließung mit Lord Derby, einem Engländer, den sie wie seinen Landsmann Lord Seymour, den sie eigentlich liebt, am Hof kennengelernt hatte. Aber die Ehe erweist sich als Betrug, Derby als skrupelloser Verführer, der sich nach dem Scheitern einer Vergewaltigung nach England absetzt. Sophie und ihre Zofe Rosina, Tochter ihres heimatlichen Pfarrers, mit der sie schon nach D. aufgebrochen war, finden Zuflucht bei Emilia, Rosinas Schwester. Nach überstandener Krankheit besinnt sich Sophie auf die „wahren Güter unseres Lebens“ (236). Um Gutes zu tun, wie sie es gelernt hat, errichtet sie eine Mädchenschule und ein „Gesindhaus“ (238). Auf einer Reise lernt sie Lady Summers kennen, eine Engländerin, die sie begeistert von ihrer Art und ihrem Engagement auf ihren Landsitz einlädt. Hier findet Sophie in Lord Rich, einem Schloßnachbarn, einen Verehrer. Doch Derby, mittlerweile mit der Nichte Lady Summers' verheiratet, fürchtet seine 
Entdeckung. Er läßt Sophie in die „schottischen Bleygebürge“ (303) entführen und schließlich, als sie sich weigert, zu ihm auf ein Landhaus nach Windsor zu kommen, einkerkern. Sie wird von ihren Wirtsleuten gerettet, glaubt aber zu sterben. Inzwischen hat Seymour die Suche nach ihr aufgenommen und in England seinen Bruder Rich getroffen. Sie finden die Vermisste, wollen auf sie verzichten, aber Sophie und Seymour kommen zusammen. Das Paar läßt sich auf Seymourhouse nieder, Rich übernimmt die Erziehung ihres zweitgeborenen Sohnes.

Sagers Die verwechselten Töchter schildert die Folgen eines Kindestausches, den Frau v. G., eine Adelige, deren Mann im Krieg den Tod fand, aus Angst vor ihrem sozialen Abstieg ins Werk setzt. Sie unterschiebt ihre einzige Tochter Klara ihrer Freundin, Frau v. Salis, als diese ihre eigene Tochter - die Mädchen sind an einem Tag geboren, tragen denselben Namen und waren Milchschwestern - nach 10 Jahren endlich zu sich an den Hof in Ma. holen will, wo sie sich als Gesellschafterin der Gräfin Crosie ein gesichertes Dasein aufgebaut hat. Schnell zeigt sich, dass Klara v. G. die falsche Klara ist. Während Klara v. Salis bei Frau v. G. in P. zurückbleibt und ohne Murren in deren florierendem Modegeschäft arbeitet, verfällt Klara v. G. in Ma. trotz der ,natürlichen' Erziehung der beiden Frauen dem höfischen Schein und seinen Versprechungen. Als ihr älterer Bruder Timon den Betrug ihrer Mutter aufdeckt, muss sie ihren Platz an Klara v. Salis abtreten. Diese heiratet Timon. Da Frau v. G. ihre Tat bereut, wird sie wieder in die Gesellschaft aufgenommen. Die falsche Klara hingegen fällt aus allen Bindungen heraus, sie stirbt, ohne Einsicht in ihre Verblendung.

Karolinens Tagebuch, ohne ausserordentliche Handlungen oder gerade so viel als gar keine handelt vom Alltag der 17jährigen Karoline, die auf dem Land lebt. Sie führt aus Lust zum Schreiben ein Tagebuch, dem sie ,alles“ (10) anvertraut und das sie gleichzeitig ihrer Schwester Nanette und ihrem gemeinsamen Schreibmeister Cyrili zur Korrektur vorlegt: um sich „Zu verbessern“ (5). Als ihre Hochzeit mit Karl v. Rieger heranrückt, fürchtet Karoline um ihr Schreiben und - schreibt immer mehr. Sie rückt in ihr Tagebuch die Briefe ihrer Freundin Eleonora von Lusani ein, die auf einer Reise entführt und in einem unzugänglichen Hause eingesperrt worden war. Hier trifft sie auf die Herzogin v. C., die ihrerseits gefangengehalten worden war, und zwar in eben dem Haus, in dem sich Eleonora befindet. Wie sie ihr erzählt, hatte sie ihr Ehemann, der Herzog von **, hierher verbracht, um die nichtstandesgemäße Ehe mit ihr zu vertuschen. Den Sohn, den sie geboren hatte, hatte er ihr weggenommen, sie selbst hatte sich erst nach 28 Jahren mit Hilfe ihrer Kammerfrau Fani aus ihrer Gefangenschaft befreien können. Karolines Heirat steht bevor, sie muss aufhören zu schreiben.

Das Geschlecht der Titelfiguren wird durch zwei Momente markiert, die Erziehung und ihren eigenen Bezug auf ihr Geschlecht. Da ist zunächst die Erziehung - erst sie macht aus den Titelfiguren „weibliche“ Figuren, und zwar nach Maßgabe der erwähnten „Geschlechtscharaktere“. Diese sind ihnen von der Natur gegeben, bedürfen aber, so die Romane, zu ihrer Entfaltung einer zeitgemäßen, Rousseau zufolge natürlichen Erziehung (Becker-Cantarino 405; Starobinski, Rousseau; Derrida). Die verwechselten Töchter stellt demonstrativ die der beiden Klaren gegenüber: Während an der falschen Klara die natürlichen, an den Bedürfnissen des Kindes nach Liebe und Verständnis ausgerichteten Erziehungspraktiken der Frau v. Salis und der Gräfin Crosie abprallen, schadet der richtigen selbst harte Arbeit nicht: „Es sind öfters die Widerwärtigkeiten und Mangel an Gemächlichkeiten viel zuträglicher, als ein immerwährendes Wohlleben und Ueberfluß; sie bringen uns zuverläßiger zu einer nothwendigen Selbsterkenntniß. Das sind die ächten Vorzüge, denen ich bey deiner Erziehung entgegen gesehen habe“ (108-9). In Karolinens Tagebuch ist die Erziehung der Titelfigur so gut wie abgeschlossen, bis auf das Schreiben, für 
das sie sich noch Anleitung verspricht. Die Sternheim stellt das Verhalten und die Vorstellungen der Eltern, ihre Erziehung und deren Auswirkungen auf die Tochter als Vorgeschichte der Handlung dar: Wie Karoline beherrscht Sophie v. Sternheim Haushaltsführung und Frauenzimmerarbeiten; hinzu kommen Philosophie, Geschichte und Sprachen sowie der Umgang mit Musik und Tanz. Sophie resümiert: „Willige Fähigkeiten, gute Beispiele und liebreiche Ausführung haben mich so gut gemacht, als tausend andere auch sein könnten, wenn sich alle Umstände zu ihrem Besten vereinigt hätten wie bei mir" (52). Aber genau dies, insbesondere ihre Vorliebe für eine einfache Lebensweise und die Sorge, Gutes zu tun, unterscheidet sie von allen anderen Frauen, wie man am Hof in D. feststellt: „Das Mädchen macht eine ganz neue Gattung von Charakter aus!“ (142) Diese „,neue“ Identität der Heldin ist das Zentrum des Romans, Charakter und weibliches Geschlecht fallen zusammen. Erst diese „Singularität“ (15), Wieland zufolge die Eigenschaft der Sternheim, löst die Handlung aus, „,worin Sie diese liebenswürdigste junge Dame in Schwierigkeiten und Umstände verwickelt sehen werden, die den schönen Plan eines glücklichen Lebens, den sie sich gemacht hatte, auf einmal zerstörten, aber durch die Probe, auf welche sie ihren innerlichen Wert setzten, ihre Geschichte für die Besten unsers Geschlechts lehrreich machen" (61). Auch Sager rückt ihre Titelfiguren als einzelne in den Blick: Die beiden Klaren, aber auch ihre Mütter werden in Situationen gestossen, in denen sie sich allein bewähren müssen; wie sie, sind Eleonora Lusani und die Herzogin vollkommen auf sich gestellt.

Dass Derby, als „Bösewicht“ (Heipcke, 25-6) Antipode der Sternheim, ihre „Singularität“ auf den Begriff bringt, ist kein Zufall. Seine Diagnose bringt das zweite Moment ins Spiel, das das Geschlecht markiert, der Bezug der Titelfiguren auf ihre Geschlechtszugehörigkeit, ihre Wahrnehmung von Geschlechtlichkeit und Sexualität, ihr Umgang mit dem eigenen und dem anderen Geschlecht. Derby spricht als Verführer, ja Libertin, der erstmals auf Widerstand stößt, wie er seinem Freund in Paris [!] klagt. Seine Rede ruft das Motiv der ,verführten Unschuld" (Petriconi) auf und benennt die Grenze, die zwischen seiner Welt und der der Titelfigur verläuft. Sophie v. Sternheim droht mehrmals einer Verführung zum Opfer zu fallen, zunächst durch den Fürsten, dann durch Derby. Sie vermag zwar ihre Unschuld zu bewahren, aber nur um den Preis, die eigene Geschlechtlichkeit zu verleugnen, nicht zuletzt durch ihren „Wunsch“, von Seymour befreit zu werden (227). Im Gegensatz dazu spielt die „verführte Unschuld“ bei Sager keine Rolle. Starobinski folgend verkörpert Timon v. G. in den Verwechselten Töchtern nicht den Verführer, sondern den Ankläger („Anklagen und verführen"): er sinnt nicht auf die Verführung der Geliebten, sondern die Aufdeckung des Betruges und die Entschädigung der Betrogenen. Umgekehrt verliebt sich der Sohn der Gräfin Crosie zwar in die falsche Klara, tut sich aber schwer, „gefällig zu werden“ (57). Als die beiden Klaren sich verlieben, verstört sie dies zwar, wirft aber selbst die falsche Klara nur aus der Bahn, weil sie an ihrer erschlichenen Identität festhält. Wie die richtige Klara vorführt, meint weibliche Unschuld bei Sager gerade nicht die Kopplung von Unschuld und Verführung, sondern die von Tugend und sozial-moralischer Bewährung, von Schuldlosigkeit im christlichen Sinne und bürgerlicher Realitätstüchtigkeit (Petriconi 32-41). Auch der ,,alte Herzog von **“ (48) in Karolinens Tagebuch hat seine Jahre als „Wollüstling“ (118) hinter sich gelassen und bereut sie wie Derby im nachhinein; sexuelles Begehren erscheint als Vergehen der Vergangenheit, treibt aber die Gegenwart nicht um.

Die verwechselten Töchter spielen die Frage nach dem weiblichen Geschlecht allein als Frage von Identität durch, im Widerstreit von Wahrheit und Betrug, Täuschung und Verstellung. Falsche Kopien, untergeschobene Doppelgänger besetzen Plätze, die ihnen nicht zukommen - Identität ist nicht automatisch gegeben, sondern austauschbar und künstlich 
(Schwarz). Erst wenn man ihr nachhilft, wenn man sie herstellt, kann sie einwandfrei festgestellt werden. Karolinens Tagebuch setzt gleichsam an diesem Befund an, zieht aber die weibliche Identität von Anfang an in Zweifel. Karoline ist weder Kopie wie die falsche Klara noch Tauschobjekt wie Sophie von Sternheim, deren Tante sie aus egoistischen Motiven an den Fürsten verkuppeln will: sie irritieren ihre Erfahrungen beim Schreiben und als Braut. Ihre Verunsicherung wiederholt sich an den beiden, durch ihr Schreiben ins Spiel gebrachten Figuren. Die an ihnen verübte anonyme körperliche Gewalt führt auf denselben Grund zurück wie die Irritationen Karolinens: eine geplante oder vollzogene Heirat. Isoliert und nur auf sich gestellt, weiß Eleonora Lusani nicht, wo und woran sie ist. Sie verdächtigt ihre Mutter, sie wie eine „Waare[n]“ (45) an den ,alten Herzog von **“ (48) zu verschachern. Die Situation der Herzogin ist noch heilloser. Einmal verheiratet, ist sie hilflos ihrem Mann und seinen Entscheidungen ausgeliefert. Auch sie sieht sich als „Ware“ (172) und „Schlachtopfer“ (122).

Ein Blick auf die Räume, in denen sich die Titelfiguren bewegen, zeigt, dass die Romane sich andererseits keineswegs mit den vom Geschlechtsunterschied hervorgebrachten Rollenzuschreibungen begnügen. Während der Begriff „Geschlechtscharakter“ beiden Geschlechtern analog zur „Dissoziation von Erwerbsund Familienleben“ (Hausen) unterschiedliche gesellschaftliche Räume zuweist, dem Mann den offenen Raum von Arbeit, Berufstätigkeit und Öffentlichkeit, der Frau den Binnenraum von Familie und Privatheit, durchqueren die Titelfiguren diese noch mühelos. Lotmans Raumsemantik erlaubt darüber hinaus, diese Räume genauer zu bestimmen. Der Vergleich zeigt wesentliche Unterschiede zwischen den Titelfiguren und verdeutlicht die Art und Weise, wie die Texte weibliche Identität, Geschlecht und Roman zusammendenken.

Lotman begreift „die räumliche Ordnung der erzählten Welt“ als „organisierendes Element“, „um das herum auch die nicht-räumlichen Charakteristika aufgebaut werden“ (Martinez und Scheffel 141); die „Sujethaftigkeit“, Lotman zufolge entscheidende Struktur narrativer Texte, hängt direkt von der Raumsemantik ab, die ein Text aufweist. Ein „Sujet“, Grundlage jeder „Sujethaftigkeit“, setzt sich aus drei „notwendigen Elementen“ zusammen: „1. ein semantisches Feld [i. e. eine erzählte Welt], das in zwei komplementäre Untermengen aufgeteilt ist; 2. eine Grenze zwischen diesen Untermengen, die unter normalen Bedingungen impermeabel ist, im vorliegenden Fall jedoch . . . sich für den die Handlung tragenden Held als permeabel erweist; 3. der die Handlung tragende Held. Aus diesen Elementen entsteht ein Sujet, wenn der Held die Grenze zwischen den beiden komplementären Teilräumen überschreitet"“ (Martinez und Scheffel 140).

Die Frage nach der Überschreitung der Grenzen, die zwischen den meist ,gegensätzlich semantisierten Teilräumen“ (Martinez und Scheffel 191) der erzählten Welt bestehen, erschließt die „Sujethaftigkeit“ von Texten und damit weitere Strukturmerkmale von Titelfiguren und Romanen. Sophie v. Sternheim wird gezwungen, das ruhige Landleben auf dem Gut der Eltern gegen die Intrigen am Hof in D. einzutauschen, hält hier aber unbeirrbar an ihren Besetzungen fest: gut steht gegen böse, vertraut gegen fremd, natürlich gegen künstlich (Martinez und Scheffel 140; Dedner). Dagegen ist Sophies Flucht aus D. freiwillig; sie beschließt den ersten Teil des Romans. Während sie fortan, im zweiten Teil, immer neue Grenzen überwindet - aus dem „einsamen unbekannten Dorf“ (210) über Vaels bei Aachen und das belgische „Spaa“ (274) in ihr geliebtes England -, rufen die selbstgewählten Räume durch ihre abgelegene ländlichfriedliche Lage die ihrer Herkunft auf. Sophie kann hier anders als am Hof - in aller Öffentlichkeit sozial tätig sein, nennt sich aber, Zeichen ihrer Verfolgung, „Madam Leidens“ (235). Aus den „schottischen Bleygebürgen“ (303), in denen Derby sie gefangengehalten hatte, rettet sie erst das Auftauchen von Rich und Seymour, an dessen Hand sie in die ländlich-lieblichen Gegenden Englands zurückkehren kann: 
... meine innerliche Stimme heißt mich meine Vermählung mit Lord Seymour als ein von dem Schicksal gegebenes Mittel ergreifen, um meiner unsteten Wanderschaft ein Ende zu machen. Und war er nicht der Mann, den sich mein Herz wünschte? Er weiß es, soll ich nun zurücke? Lord Rich, fürchte ich, würde an seinen Platz eintreten wollen (341).

Die Titelfigur überschreitet zwar diverse Räume und Grenzen, kehrt aber an den idealen Ort und in die Welt zurück, die sie kennt und liebt und die überdies in England liegen, immer schon Ziel ihrer „Begierde“ (199) (Maurer). Die Wertungen, mit denen sie ausgezogen war, behalten ihre Gültigkeit; ihre Erfahrungen mit „Schwierigkeiten” (61) werden „wieder rückgängig gemacht und damit aufgehoben“ (Martinez und Scheffel 142) - die „domestic fiction“ (Kontje) ist am Ziel. Der Text bestätigt „die klassifikatorische Ordnung der erzählten Welt", man kann ihn mit Lotman „restitutiv“ (Martinez und Scheffel 142) nennen.

Dasselbe gilt für Die Verwechselten Töchter. Auch hier stehen Räume einander gegenüber, so die Stadt P. und der Hof in Ma., das Modegeschäft und das adelige Palais der Gräfin Crosie. Während die falsche Klara den Wechsel an den Hof nicht übersteht, weil sie seine Wertungen übernimmt, ist die richtige Klara überall auf die gleiche Weise zu Hause. Anstatt wie Sophie v. Sternheim zu Ortswechseln und Grenzüberschreitungen gezwungen zu sein, erlebt sie dank Timons Aufklärungsarbeit nur ihre soziale Rehabilitation. Dass die „Ordnung der erzählten Welt" bekräftigt wird, zeigt auch ein Blick auf die Mütter: Frau v. G.s Reue bahnt ihr den Weg an den unter dem Einfluß der Gräfin Crosie und Frau v. Salis geläuterten Hof; Frau v. Salis nimmt zwar einen Dienst an, erhält aber ebenfalls ihren angestammten Platz in der Gesellschaft zurück.

Im Unterschied zu diesen Figuren überschreitet die Herzogin in Karolinens Tagebuch die ihr gesetzten Grenzen auf grundlegend andere Weise. In dem Maße, in dem ihr die Begegnungen mit sich selbst - in ihrem Spiegelbild und ihrem eigenhändig verfaßten, „meiner Geschichte ähnliche[n] Roman“ (137), ihrem Porträt als Schriftstellerin und ihrer Melancholie - Bilder von ihrem Ich zuspielen, findet sie zu ihrer Identität: Sie hebelt die Vormundschaft ihres Gemahls aus und befreit sich aus ihrem Gefängnis. Ihr Aufbruch in die städtischbürgerliche Welt ist buchstäblich „revolutionär“ (Lotman): Sie überwindet nicht nur Gefangenschaft und Melancholie, sondern auch die ihr auferlegte Unterdrükkung und die Schranke zwischen Innen und Außen. Dagegen ist Eleonora Lusanis Verbleib ungewiß. Und auch Karoline vermag sich den mit dem Ehestand gesetzten Normen nicht zu entziehen: „Hauswirthschaft und Sorgen zeigen sich mir fast in einer fürchterlichen Gestalt” (303). Während ihr diese ,Grenze' unüberwindlich erscheint, überschreitet sie sie in einem anderen Sinne, und zwar indem sie an ihrer „Lust zum Schreiben“ (6) festhält und sich selbst beim Schreiben imaginiert:

Wenn ich so fortfahre, so kann ich mit der Zeit nicht allein abschreiben, sondern auch selbst Romanen erfinden. Ich will mir indessen nur Stoffe dazu sammeln.

Aus dieser Geschichte kann ich zwar nach meinem Geschmacke, vielleicht auch nach dem deinigen [Nanettes, H. M.] nicht viel erhaschen: aber warte, wenn ich diese Mattigkeiten werde vollendet haben, dann werde ich etwas Anziehenderes machen, wo etwas mehr von den Handlungen zu lesen seyn wird. Ein paar Helden habe ich schon im Vorrath, die ihre Prinzeßinnen von ihren Verfolgern erretten ... (256)

Jedes Weiterschreiben eröffnet den Imaginationsraum für eine andere, neue Identität, wird wie für Werther zum „Vertauschungsexperiment mit der eigenen Identität“ (Erhart 341). Noch ihr letzter Brief an ihre Schwester zeigt, dass der neue Personenstand ihr das Schreiben keineswegs austreibt:

Und jetzt soll ich schon aufhören zu schreiben, soll denn unsere Hochzeit so einfach weg ablaufen; nichts ausserordentliches vorfallen? Kein Zweykampf, keine Prüfung der 
standhaften Tugend, keine Verleumdung eines falschen Freundes, die das Hochzeitsfest verschieben könnten; soll kein Onkel oder Tante sterben, um uns reich zu machen? $\mathrm{O}$ was wird das für eine matte Geschichte von uns beyden werden? (302)

Karolinens Einwilligung in die Heirat ist zwar die Annahme der Rolle der Ehefrau, im Festhalten am Schreiben aber gelingt auch ihr die ,revolutionäre“ Überschreitung.

\section{Weibliches Geschlecht und Gattung: Polyperspektive versus mise en abyme}

Die Darstellungsformen, die La Roche und Sager wählen, unterstreichen die Unterschiede zwischen den Texten ihrerseits. Die Sternheim „kommt dem englischen Muster des empfindsamen Romans formal und inhaltlich am nächsten“, notieren D'Aprile und Siebers (179). In der Nachfolge der Briefromane Samuel Richardsons (Beebee; Becker-Cantarino 403) wird das Geschehen in Briefen aus der wechselnden Perspektive der Beteiligten geschildert, ist aber durch Weglassen der Gegenbriefe und die Einführung der anonymen „Freundin“, die „Original-Papiere und ander[e] zuverläßige Quellen“ (Titel) zusammengetragen hat und nun einer Freundin vorlegt, weitgehend gestrafft. Diese gibt sich zu Beginn als Rosina, Zofe des Fräuleins, zu erkennen. Nach der Vorgeschichte - Rosina schildert die Liebesgeschichte der Eltern und Sophies Erziehung - läßt sie die „Originalbriefe, oder Abschriften“ (61) zu Wort kommen und schaltet sich nur noch ein, wenn sie Lücken ,in den Papieren ausfüllen“ (302) oder die Handlung erläutern will. Anders als der Herausgeber Wieland verbleibt sie innerhalb der erzählten Welt. Ihr Mitleid mit Sophie, die sie aus Schuldgefühl - „daß ich unglücklicherweise . . . zu allem half“ (198) - bis nach England begleitet, wird zur Leserlenkung, unterstützen ihre Kommentare doch aus weiblicher Perspektive die unmittelbare Teilnahme an den „Gedanken und Empfindungen der Briefschreiberin“ (Häntzschel 329), wie die Reaktion der wohl berühmtesten SternheimLeserin bezeugt. Gemeint ist Caroline Flachsland (1750-1809), die Verlobte Johann Gottfried Herders (1744-1803): „Ich habe indeßen auch [die] Geschichte der Fräulein von Sternheim gelesen, mein ganzes Ideal von einem Frauenzimmer! sanft, zärtlich, wohlthätig, stolz und tugendhaft. und betrogen. Ich habe köstliche, herrliche Stunden beym Durchlesen gehabt. ach, wie weit bin ich noch meinem Ideal von mir selbst weg! Welche Berge stehn gethürmt vor mir!“ (365) Während Sophie v. Sternheim ihren Fall („Ich bin in den Staub erniedriget; auf der Erde liege ich, ... “ [228]) durch gute Taten überwindet (Touaillon 104-108), kehrt die Leserin ihre Metaphern gegen sich und die eigene Zukunft: ,,ach! ach, ich werde im Staub und in der Asche bleiben!“ (365) Der polyperspektivische Briefroman nimmt die „,neue Gattung von Charakter" gleichsam immer wieder neu in den Blick, bis ihre Identität endgültig fixiert ist und Leserin und Leser nicht nur zur Identifikation, sondern zur Überbietung reizt.

Die Romane Sagers stellen im Gegensatz dazu die Titelfiguren in sich widersprüchlich dar und erproben Darstellungsformen, die Geschlechtsrolle und Leseridentifikation gleichermaßen unterlaufen. Die verwechselten Töchter ersetzt die Polyperspektivität durch die Rahmung des Erzählten, die in einer Figur, der richtigen Klara, ihr Zentrum hat. Der anscheinend zeitdeckend erzählte Kindestausch erweist sich in Briefen einer alten Freundin gegenüber als Rückblick:

Sie begehren einen schriftlichen Aufsatz von dem Leben meiner unglücklichen Freundin von mir, weil, so wie Sie sagen, eine Geschichte in der Erzählung mehrentheils die Hälfte ihres Werthes verlöre, besonders wenn gewiße Nebenumstände, die öftermalen die Hauptsache in eine besseres Licht setzen müssen, darbey vergessen werden. Wissen Sie aber Madame! Daß ich bey einem schriftlichen Aufsatze selbst einbüße? (1) 
Die sich im anschließend dargebotenen Briefwechsel mit Klara v. G. langsam aufbauende Spannung, wer die richtige Klara ist, ist nur fiktiv, ist doch von Anfang an klar, dass es um die Geschichte „meiner unglücklichen Freundin“ geht, die der falschen Klara. Dies weist die Erzählerin, selber Figur ihrer Erzählung, automatisch als richtige Klara aus. Dass sie sich nur wenig später als „Ältere“, zuerst geborene bezeichnet, verleiht ihr zusätzlich Autorität. Wiederkehrende Anspielungen, etwa in den Attributen der Frau v. G. oder in der Figurenrede, die in den Briefen wiedergegeben wird, halten die Wahrheit von Anfang an präsent. So schildert Klara v. G. ihr Eintreffen in Ma. ihrer Freundin mit den Worten:

\begin{abstract}
Meine Mutter empfieng mich auf das zärtlichste, und rufte scherzend aus: „Mädgen! Ich muß mich an meiner Freundinn versehen haben, du siehst mehr ihr, als mir gleich. Doch ich weis ihr Dank dafür, sonst wärest du nicht so schön. Nun liebste Mama! fiel ich ihr betrübt in die Rede , wäre ich Ihnen denn nicht so lieb, wenn ich der Frau v. G. nicht ähnlich sähe? „Nicht doch, versetzte sie, du würdest mir in allen Gestalten lieb seyn, aber noch um so lieber, weil du mich an sie erinnerst. . . . Gut Mama! indem ich ihr die Hand küste, ich kann Sie dagegen versichern, daß meine liebe Freundinn Klara G. Ihnen gleichfalls vollkommen ähnlich ist. Sie sahe mich starr an, sagte weiter nichts, und küste mich. Nach einigen Fragen, die die Frau v. G. betraffen, gieng sie zu der Gräfinn, um, wie es schiene, ihr meine Ankunft zu melden. Nach einer ziemlich langen Unterredung kam sie endlich wieder . . (47-48)
\end{abstract}

Derart ausgestreute Hinweise auf den Kindestausch laufen als Subtext mit. In dem Maße, in dem Klara v. Salis als wahre Klara inthronisiert wird, wird die falsche Klara ausgestrichen.

Karolinens Tagebuch verwirft die lineare, von Beginn an auf das Tableau des Schlusses zulaufende Darstellung der Verwechselten Töchter grundsätzlich, wie Baldwin an den „metafictional considerations“ (142) ebenso darlegt wie an den eingerückten Geschichten. Schon der Titel zeigt an, dass der Roman die Vertauschung nicht als Sujet durchspielt, sondern als Darstellungsform. Das angekündigte Tagebuch ist mehrfach gestuft, vom Tagebuch über ohne ausserordentliche Handlungen bis hin zu oder gerade so viel als gar keine. Die Stufung erscheint als Abstufung, als Widerruf, was die typographische Gestaltung noch unterstreicht. Die Schriftgröße wird von Stufe zu Stufe kleiner, bis der letzte, der dritte Teil des Titels, beinahe unkenntlich ist. Die Vorrede spitzt die Herabsetzung, die „Nichtung“ weiter zu: „Wie, meine lieben Leserinnen, Sie haben das Titelblatt gelesen . . . wollen sie mit Nichts - und was ist ein Tagebuch ohne ausserordentliche Handlungen anderst - ihre Zeit versplittern?“ (unpag.). Erst das „Nichts“ (Baldwin, 143ff; Wögerbauer) befreit die Autorin von überkommenen Traditionen und der aktuellen Gattungsdiskussion und erlaubt, ihre Lust und ihren Anspruch auf literarische Produktivität umzusetzen: „Denn je mehr man mich von etwas abhält, umso begieriger bin ich darauf.“ (unpag.) Sager spielt mit der ,Stufung' des Erzählten und schafft auf diese Weise ein Werk. Ebenso erlauben die Erzählungen, die Karoline in ihr Tagebuch einspeist, ihre Rolle als Ehefrau anzunehmen und gleichzeitig deren Anforderungen $\mathrm{zu}$ entfliehen. Es geht nicht um eine Flucht in eine fiktive Welt. Mit demselben Trick, mit dem die Titelfigur aus der Darstellung ihres Alltags in die Fiktion wechselt, wechselt sie auch wieder zurück: Das Personal der Fiktion tritt im Tagebuch, in der Realität auf. Der junge Herzog erscheint als Initiator der Lusanischen Geschichte, die Lusanische Geschichte als Stoff des Tagebuches, das Tagebuch als „Nichts“. Das den Erzählungen aller Stufen gemeinsame Sujet, die weibliche Identität bzw. deren Disponibilität, erlaubt ihre jederzeitige Vertauschung. Die Spiegelung der Geschichten ineinander, ihre wechselseitige Kommentierung und Ironisierung, die beständige Rahmenüberschreitung und Aufhebung der Grenzen zwischen Tagebuch, Lusanischer Geschichte und Geschichte der Herzogin unterläuft gerade den Zusammenhang von Gattung, Geschlecht und Autorschaft, den Wielands Vorrede propagiert. Die paradoxe Konstruktion einer mise en abyme (Baldwin 142) hebelt Wielands Konstruktion weiblicher Autorschaft aus: Sager deckt die 
Produktionsbedingungen einer Autorin auf und setzt sie im selben Moment ästhetisch um, namentlich in ihrem zweiten Roman. Auch wenn Karolinens Tagebuch ausserhalb Böhmens, in Friedrich Nicolais (1733-1811) Allgemeiner deutscher Bibliothek, rezensiert wurde (Meise), wurden Sagers Romane im Gegensatz zur Sternheim kaum wahrgenommen. Zunächst setzte sich das Modell La Roches durch, zumindest bis zu seiner Abwertung durch den Siegeszug des Bildungsromans. Im Rückblick allerdings erweist sich das Modell Sagers als missing link für heutige Diskussionen um Gattung, Geschlecht und Autorschaft.

\section{Zitierte Werke}

Baldwin, Claire. The Emergence of the Modern German Novel. Christoph Martin Wieland, Sophie von La Roche, and Maria Anna Sagar. Rochester: Camden House, 2002 (Studies in German Literature, Linguistics, and Culture).

Becker-Cantarino, Barbara. Nachwort. Geschichte des Fräuleins von Sternheim. Ed. Barbara Becker-Cantarino. Stuttgart: Reclam, 1983. 381-415.

Beebee, Thomas O. Clarissa on the Continent. Translation and Seduction. University Park: Pennsylvania UP, 1990.

D’Aprile, Iwan-Michelangelo, und Siebers, Winfried. Das 18. Jahrhundert. Zeitalter der Aufklärung. Berlin: Akademie, 2008.

Dedner, Burghard. Topos, Ideal und Realitätspostulat. Studien zur Darstellung des Landlebens im Roman des 18. Jahrhunderts. Tübingen: Niemeyer, 1969 (Studien zur deutschen Literatur, 16).

Derrida, Jacques. Grammatologie. Frankfurt am Main: Suhrkamp, 1974.

Dawson, Ruth. The Contested Quill. Literature by Women in Germany, 1770-1800. Newark: U of Delaware Press, 2002.

Erhart, Walter. „Werther und Wieland“. Deutsche Vierteljahrsschrift für Literaturwissenschaft und Geistesgeschichte 66.2 (1992): 333-361.

Fleig, Anne und Meise, Helga. „Das Geschlecht der Innovation: Bedeutung und Reichweite der Verknüpfung von Gattungs- und Geschlechterdiskurs bei Gellert, Sulzer und Wieland“. Das Achtzehnte Jahrhundert 29.2 (2005): 159-179.

Frick, Werner. Providenz und Kontingenz. Untersuchungen zur Schicksalssemantik im deutschen und europäischen Roman des 17. und 18. Jahrhunderts. 2 Teile. Tübingen: Niemeyer, 1988 (Hermaea, 55).

Goethe, Johann Wolfgang von. Wilhelm Meisters Lehrjahre. Werke. Ed. Erich Trunz. München: Beck, 1982. Bd. 7.

Häntzschel, Günter. Nachwort. Geschichte des Fräuleins von Sternheim. Ed. Günter Häntzschel. München: Winkler, 1976 (Die Fundgrube). 301-337.

Hausen, Karin. „'Die Polarisierung der „Geschlechtscharaktere’ - eine Spiegelung der Dissoziation von Erwerbs- und Familienleben“. Sozialgeschichte der Familie in der Neuzeit Europas. Ed. Werner Conze. Stuttgart: Klett, 1977. 363-384.

Heipcke, Corinna. Autorhetorik. Zur Konstruktion weiblicher Autorschaft im ausgehenden 18. Jahrhundert. Frankfurt am Main: Lang, 2002 (Studien zur Neueren Literatur, II).

Heuser, Magdalene. ,,Ich wollte dieß und das von meinem Buche sagen, und gerieth in ein Vernünfteln'. Poetologische Reflexionen in den Romanvorreden“. Untersuchungen zum Roman von Frauen um 1800. Ed. Helga Gallas und Magdalene Heuser. Tübingen: Niemeyer, 1990 (Untersuchungen zur deutschen Literaturgeschichte, 55). 52-66.

Honegger, Claudia. Die Ordnung der Geschlechter. Die Wissenschaften vom Menschen und das Weib. Frankfurt am Main, New York: Campus, 1991.

Jirku, Brigitte. „Wollen Sie mit Nichts . . . ihre Zeit versplittern?“ Ich-Erzählerin und Erzählstruktur in von Frauen verfaßten Romanen des 18. Jahrhunderts. Frankfurt am Main: Lang, 1994 (Forschungen zur Literatur- und Kulturgeschichte, 39).

Kohl, Katrin. „Metaphorik des Schreibens und Lesens um 1770“. Gefühlskultur in der bürgerlichen Aufklärung. Ed. Achim Aurnhammer et al. Tübingen: Niemeyer, 2004. 23-47.

Kontje, Todd. Women, The Novel, and The German Nation 1771-1871. Cambridge: Cambridge UP, 1998.

[La Roche, Sophie von]. Geschichte des Fräuleins von Sternheim. Von einer Freundin derselben aus Original=Papieren und andern zuverläßigen Quellen gezogen. Herausgegeben von C. M. Wieland. 2 
Theile. Leipzig, bey Weidmanns Erben und Reich. 1771. Ed. Barbara Becker-Cantarino. Stuttgart: Reclam, 1983.

Laqueur, Thomas. Auf den Leib geschrieben. Die Inszenierung der Geschlechter von der Antike bis Freud. Frankfurt am Main: Campus, 1992.

Loster-Schneider, Gudrun. Sophie La Roche. Paradoxien weiblichen Schreibens im 18. Jahrhundert. Tübingen: Gunter Narr, 1995 (Mannheimer Beiträge zur Sprach- und Literaturwissenschaft, 26).

Lotman, Jurij M. Die Struktur literarischer Texte. Übersetzt von Rolf-Dietrich Keil. München: Fink, 1972.

Martinez, Matias und Scheffel, Michael. Einführung in die Erzähltheorie. München: Beck, 1999.

Maurer, Michael. Aufklärung und Anglophilie in Deutschland. Göttingen: Vandenhoeck \& Ruprecht, 1987 (Veröffentlichungen des Deutschen Historischen Instituts London, 19).

Meise, Helga. Nachwort. Die verwechselten Töchter, eine wahrhafte Geschichte in Briefen entworfen von einem Frauenzimmer. Karolinens Tagebuch, ohne ausserordentliche Handlungen oder gerade so viel als gar keine. Hildesheim: Olms, 2013 (Frühe Frauenliteratur). Im Druck.

Meise, Helga. „Von der , unsichtbaren’ zur ,sichtbaren’ Geschichte. Zur Prager Herkunft der Schriftstellerin Maria Anna Sager, geb. Roßkoschny (1719-1805)“. Cornova 1.1 (2010): 51-66.

Nörtemann, Regina. „Die Begeisterung eines Poeten in den Briefen eines Frauenzimmers. Zur Korrespondenz der Caroline Christiane Lucius mit Christian Fürchtegott Gellert“. Die Frau im Dialog. Ed. Anita Runge und Lieselotte Steinbrügge. Stuttgart: Metzler, 1991. 13-33.

Petriconi, Hellmuth. Die verführte Unschuld. Bemerkungen über ein literarisches Thema. Hamburg: Cram, de Gruyter, 1953 (Hamburger Romanistische Studien, A, 38).

S[ager], M[aria] A[nna]. Karolinens Tagebuch, ohne ausserordentliche Handlungen oder gerade so viel als gar keine. Geschrieben von M. A. S. Prag, bey Wolfgang Gerle, 1774.

[Sager, Maria Anna]. Die verwechselten Töchter, eine wahrhafte Geschichte in Briefen entworfen von einem Frauenzimmer. Prag bey Wolfgang Gerle. 1771.

Schlimmer, Angelika. Romanpoetik und Weiblichkeitsdiskurs. Zur Bedeutung der Kategorie gender im Romanverständnis von Therese Huber und Johanna Schopenhauer. Königstein/Ts.: Helmer, 2001

Schlimmer, Angelika. „Der Roman als Erziehungsanstalt für Leser. Zur Affinität von Gattung und Geschlecht in Friedrich von Blanckenburgs Versuch über den Roman’ (1774)“. Das Achtzehnte Jahrhundert 29.2: 209222.

Schön, Erich. „Weibliches Lesen: Romanleserinnen im späten 18. Jahrhundert“. Untersuchungen zum Roman von Frauen um 1800. Ed. Helga Gallas und Magdalene Heuser. Tübingen: Niemeyer, 1990 (Untersuchungen zur deutschen Literaturgeschichte, 55). 20-40.

Schwarz, Hillel. The Culture of the Copy. Striking Likenesses, Unreasonable Facsimiles. New York: Zone Books, 1996.

Starobinski, Jean. „Anklagen und verführen als typische Gesten der Aufklärung. Gespräch mit Marcel Lepper und Ulrich Raulff“. Frankfurter Allgemeine Zeitung. 03.11.2010: N4.

Starobinski, Jean. Jean-Jacques Rousseau. La transparence et l'obstacle. Paris: Gallimard, 1971.

Stern, Guy. „Wieland als Herausgeber der Sternheim“. Christoph Martin Wieland. Nordamerikanische Forschungsbeiträge zur Wiederkehr seines 250. Geburtstages 1983. Ed. Hansjörg Schelle. Tübingen: Niemeyer, 1984. 195-208.

[Sulzer, Johann Georg]. „Vorrede“. Anna Louisa Karsch, Auserlesene Gedichte. Faksimiledruck nach der Ausgabe von 1764. Mit einem Nachwort von Alfred Anger. Stuttgart: Metzler, 1966 (Deutsche Neudrucke. Reihe Texte des 18. Jahrhunderts). VIIXXVI.

Touaillon, Christine. Der deutsche Frauenroman des 18. Jahrhunderts. Wien, Leipzig: Braumüller, 1919.

Wieland, Christoph Martin. „An D. F. G. R. V.******“. Geschichte des Fräuleins von Sternheim. Stuttgart: Reclam, 1981. 9-17.

Wirth, Uwe. Die Geburt des Autors aus dem Geist der Herausgeberfiktion. Editoriale Rahmung im Roman um 1800. München: Fink, 2008.

Wögerbauer, Michael. „Romany ,o nicem’. Prazská spisovatelka Maria Anna Sagarová (1729-1805) a její

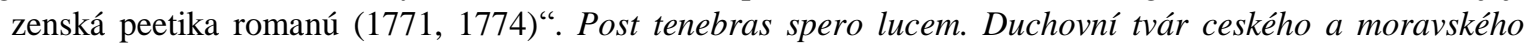
osvícenství. Ed. Jaroslav Lorman und Daniela Tinková. Praha: Casablanca, 2008. 393-405. 